\title{
Über den Einfluß von Sauerstoff auf die Empfindlichkeit photographischer Schichten
}

\author{
Von Helmer Bäckström \\ Aus dem Photographischen Institut der Kgl. Technischen Hochschule zu Stockholm \\ (Z. Naturforschg. 6 a, 377-381 [1951]; eingegangen am 21. Mai 1951) \\ John Eggert zum 60. Geburtstage gewidmet
}

\begin{abstract}
In den Poren der Gelatineschicht eines Filmes ist Luft absorbiert, deren Sauerstoffgehalt die Empfindlichkeit der Schicht vermindert. Nach gleichen Expositionen ist deshalb die Schwärzung der in Luft exponierten Schicht geringer, als wenn die Schicht evakuiert oder mit reinem Stickstcff gesättigt ist. Dies könnte durch Brombildung infolge der Gegenwart von Sauerstoff in der $\mathrm{KBr}$-Hülle der Bromsilberkörner verursacht werden; die Bromatome würden dann Phototlektronen einfangen und dadurch die Lichtausbeute verschlechtern. Die Schwärzungszunahme in Stickstoff und Vakuum scheint bei Korntiefenentwicklung besonders groß zu sein. Auch während der Stabilisierung des latenten Bildes zwischen Exponierung und Entwicklung scheint Sauerstoff eine Wirkung auszuüben.
\end{abstract}

$\mathrm{W}$ ährend der vierziger Jahre waren wir lebhaft mit einem photographischen Druckeffekt beschäftigt $^{1}$. 1944 und 1946 untersuchte dabei einer meiner damaligen Mitarbeiter, J. B a s berg, die Druckabhängigkeit des Herschel-Effektes, worüber einige kurze Mitteilungen publiziert worden sind ${ }^{2}$. Im $\mathrm{Zu}-$ sammenhang hiermit wurden von $\mathrm{B}$ a s berg und mir auch einige Versuche über den eventuellen Einfluß des Sauerstoffes der Luft auf den HerschelEffekt und die Eigenempfindlichkeit der photographischen Schicht ausgeführt. Die Resultate dieser Versuche schienen uns interessant; andere Untersuchungen haben uns jedoch gehindert, dieselben weiter zu verfolgen. Jetzt haben wir aber dieses Thema zusammen mit E. Fag e rlund wieder aufgegriffen und wir wollen vorläufig einige kurze Angaben darüber machen.

Die Erwägungen, die zu unseren ersten Versuchen über den Einfluß des Sauerstoffes führten, gingen von der Theorie Gurney und Mott's aus ${ }^{3}$. Nach dieser Theorie werden die vom Licht in dem Bromsilberkorn erzeugten Elektronen von Keimen und anderen Störstellen eingefangen, oder sie können zu den Bromatomen zurückkehren, aus welchen sie herausgeschleudert worden waren. Diese Wiedervereinigung

1 H. B ä ckström, Svenska Vet. Akad. Arkiv f. matem., astronomi o. fysik $35 \mathrm{~A}, \mathrm{Nr} .20,1-36$ [1948]; H. Bäckström u. O. A d els ohn, ebenda $35 \mathrm{~A}$, Nr. 21, 1-40 [1948]; auch H. B ä ckström, C. A. Bruno u. M. Müller, The Svedberg Festschrift, Upsala und Stockholm 1944, S. 65-81. H. B ä c k s t r ö m in J. W. Mitchell, Photographic Sensitivity (Conference at Bristol) 1951.

von Elektronen und Bromatomen spielt wohl im allgemeinen eine unwesentliche Rolle, wird aber beim Herscheln bei niedrigen Temperaturen $\left(-190^{\circ} \mathrm{C}\right)$ von großer Bedeutung ${ }^{4}$. Die Bromatome, die gewöhnlich rasch nach der Kornfläche diffundieren, um dort von der Gelatine aufgenommen zu werden, sind nämlich bei den genannten niedrigen Temperaturen im Kristallgitter festgefroren. Betrachten wir jetzt die Desensibilisatoren, so erklärte schon Lüppo-Cramer die Empfindlichkeitsverminderung durch dieselben mit ihren Oxydationswirkungen. Ich interpretierte dies dadurch, daß ich eine Oxydierung von $\mathrm{Br}$-Ionen der $\mathrm{KBr}$-Hülle an den Bromsilberkörnern annahm. Das latente Bild wird ja von Desensibilisatoren nicht angegriffen, dagegen wird das Aufkommen von neuem Bildsilber stark gehemmt. Nach meiner Meinung würde diese Wirkung daher rühren, daß die durch Belichtung hervorgebrachten neuen Elektronen sich großenteils mit Br-Atomen an der Kornfläche verbinden und nicht den Keimen zuteil werden. So betrachtet, scheint es nun auch möglich,

2 H. B äckström u. J. B a s berg, J. opt. Soc. America 37, 891-898 [1947]; H. B ä c k s trö m u. O. Adels ohn, Svenska Vet. Akad. Arkiv f. matem., astronomi o. fysik 35 A, Nr. 21 [1948]; H. B ä c k s t r ö m in J. W. Mitchell, Phot. Sensitivity (Conference at Bristol) 1951.

3 R. W. Gurney u. N. F. Mott, Proc. Roy. Soc. [London], Ser. A 164, 151 [1938]; N. F. M o t t, Photographic J. 78, 286 [1938]; N. F. M o t t u. R. W. G u r $\mathrm{n}$ e y, Electronic Processes in Ionic Crystals, Oxford 1940.

4 J. H. W e b b u. C. H. Evans, Trans. Faraday Soc. 35, 445 [1939]. 
daß die Bromsilberkörner schon normalerweise eine kleine Menge freien Broms an den Kornflächen absorbiert halten. Dieses würde durch Einwirkung des Luftsauerstoffes, der die Poren der Gelatineschicht füllt, auf die dünne $\mathrm{KBr}$-Hülle der Bromsilberkörner gebildet werden. Hierdurch würde eine kleine Verminderung der Empfindlichkeit schon normalerweise vorhanden sein. Durch Entfernen der Luft aus der Schicht würde es deshalb möglich sein, diese Verminderung zu beseitigen. Dies könnte z. B. durch Evakuieren geschehen; auch könnten die Poren nachher mit einem indifferenten Gas, beispielsweise Stickstoff, gefüllt werden. Beidenfalls würde man eine größere Empfindlichkeit bekommen und gleichzeitig würde auch die Tendenz der Schicht, bei Rotbeleuchtung einen Herschel-Effekt zu geben, vermindert werden.

Unsere allerersten Versuche in der Mitte der vierziger Jahre schlossen sich ja an unsere Arbeiten über den Einfluß von hohen Drucken auf den Herschel-Effekt an. Der Druckapparat war sehr groß und konnte nicht in einer Vakuumkammer eingeschlossen werden. Wir verfuhren deshalb in folgender Weise. Die Filme wurden in einen Stahlkasten eingelegt und dieser mittels einer Ölpumpe bis etwa $0,1 \mathrm{~mm}$ evakuiert. Die Gelatineschicht hält ihre Luft ziemlich fest und gibt sie sehr langsam ab. Die Filmstreifen wurden deshalb etwa 24 Stdn. im Vakuum gelassen; danach wurde durch Pyrogallol gereinigter Stickstoff in die Kammer geleitet, so daß der Gasdruck sich in Übereinstimmung mit dem Außendruck einstellte; die Filme blieben 24 Stdn. unter Stickstoff. Alsdann wurden sie schnell herausgeholt, in den Druckapparat gebracht und unmittelbar exponiert. Zum Vergleich wurden auch Filmstreifen in Luft von gewöhnlichem Druck in demselben Apparat exponiert. Die Entwicklung geschah dann in dem DIN-Entwickler während 5 Min. und bei konstanter Temperatur.

Diese ersten Versuche wurden mit Gevaert-Dipos-Film ausgeführt und bezogen sich zunächst auf den HerschelEffekt. Die Filmstreifen wurden in blauem Licht verschiedene Zeiten (0,3 bis 2,75 Min.) exponiert und dann halbiert. Der eine Teil wurde evakuiert und wie oben mit Stickstoff behandelt; der andere wurde ebenso lange in Luft von gewöhnlichem Druck verwahrt. Dann wurden die beiden Teile 8 Min. durch ein Rotfilter (Absorptionsgrenze bei $7300 \AA$ ) exponiert. Auch die Kontrollstreifen wurden in gleicher Weise behandelt, jedoch ohne Rotbelichtung.

Abb. 1 zeigt die Resultate. Die Kurve A bezieht sich auf blau-exponierte Filmproben, die zwischen der Exponierung und der 48 Stdn. später erfolgten Entwicklung in Luft von gewöhnlichem Druck aufbewahrt wurden. Kurve B bezieht sich auf Filme, die in gleicher Weise exponiert, aber dann 24 Stdn. im Vakuum und 24 Stdn. unter Stickstoff verwahrt wurden. Bei der Kurve C sind die Filmstreifen blau- exponiert, dann 48 Stdn. in Luft aufbewahrt und zuletzt 8 Min. rot-belichtet worden. Bei der Kurve D schließlich wurden die Filmstreifen zwischen der Blauexponierung und der Rotexponierung 24 Stdn. in Vakuum und 24 Stdn. in Stickstoff verwahrt. Wie ersichtlich, liegen die Schwärzungskurven B und D links von den Kurven A bzw. C. Man könnte den Befund auch so ausdrücken, daß das Entfernen der Luft eine Zunahme der Schwärzung mit sich bringt.

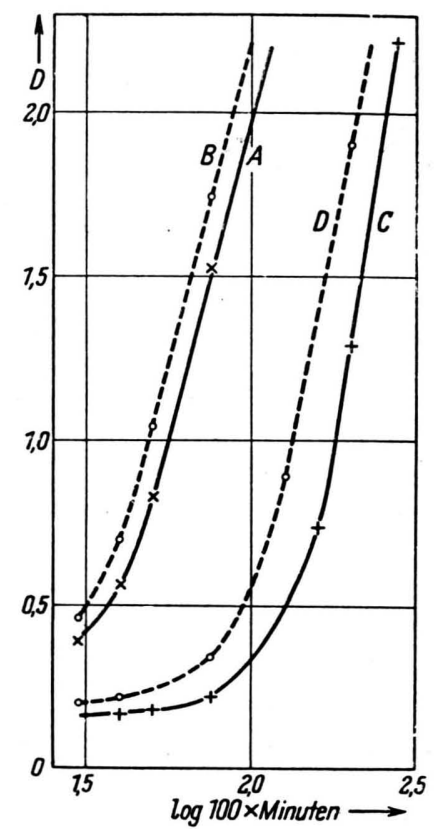

Abb. 1. Schwärzungskurven (Zeitskala) f. Gevaert - Dipos Film. Die Filmproben wurden in Luft mit blauem Licht exponiert, B und D danach evakuiert (24 Stdn.) und unter Stickstoff verwahrt (24 Stdn.). Vor der Entwicklung wurden die Filmproben $\mathrm{C}$ und D rotbelichtet.

Wenn wir zunächst die Kurven A und B betrachten, so sind die entsprechenden Filmproben in beiden Fällen' vollkommen gleich exponiert und zwei Tage danach entwickelt worden. In der Zwischenzeit, während welcher sich das latente Bild in Stabilisierung befand, wurden jedoch die Probestücke A in Luft von atmosphärischem Druck, die Filmproben B dagegen im Vakuum bzw. unter Stickstoff verwahrt. Es zeigte sich dabei, daß die Gegenwart von Luft während des Stabilisierungsprozesses eine deutliche Schwärzungsabnahme verursacht, die im Maximum 0,66 beträgt. Eine solche scheint bisher nicht beobachtet worden zu sein. Nach meiner Arbeitshypothese sollte dieser Effekt daher rühren, daß bei dem Überführen von Silber zwischen den verschiedenen Keimen ein Teil des Silbers dadurch verloren geht, daß freigegebene 
Elektronen von freiem Brom an der Kornfläche gebunden werden. Beiläufig sei bemerkt, daß die verschiedenen Meßpunkte längs der beiden Kurven durch voneinander unabhängige Versuche bestimmt wurden. Trotzdem ist die Regelmäßigkeit sehr überzeugend; die Schwärzungen der vakuumbehandelten Probestreifen sind durchgehend höher als die Schwärzungen der luftbehandelten Probestreifen mit derselben Expositionszeit. Wahrscheinlich würde die Differenz noch größer sein, falls die Proben sich schon bei der Exposition in Luft bzw. im Vakuum befunden hätten.

Wenn wir jetzt die Kurve A mit der Kurve C bzw. D vergleichen, so geht deutlich hervor, daß der GevaertDipos-Film für das Herscheln sehr empfindlich ist. Bei den hier vorkommenden Rotbelichtungen wurden die Schwärzungen der Felder mit niedrigen und mittelgroßen Dichten fast vollkommen vernichtet. Die maximale Schwärzungsabnahme durch die Rotbelichtung kann deshalb erst bei größeren Originalschwärzungen (etwa 2,5-3,0) wahrgenommen werden. Die maximale Differenz zwischen den Kurven A und C war dabei 2,03 und zwischen A und D 1,19. Wir haben die Verhältnisse bis zu den Originaldichten 4 verfolgt, aber in Abb. 1 nur den wichtigsten Teil bis etwa $D=2$ aufgenommen; die genannte Differenz sinkt wieder bei größeren Dichten.

Dieser Vergleich scheint zu zeigen, daß der Herschel-Effekt kleiner wird, wenn die Luft während der Rotbelichtung entfernt ist; die Schwärzungsdifferenz wurde ja von maximal $\Delta D=2,03 \mathrm{zu} \Delta D=1,19$ herabgesetzt. Dieser Effekt würde somit ziemlich groß sein, und es scheint zunächst eigentümlich, daß $\mathrm{Blau}$ und $\mathrm{Wambacher}{ }^{5}$ während ihrer vielen Versuche über den Einfluß von Luft auf den Desensibilisierungsprozeß denselben nicht beobachtet haben. Sie erklären nämlich mehrmals, daß sie nur bei Gegenwart von Desensibilisatoren - besonders Pinakryptolgelb - einen Einfluß der Luft bemerkt hatten. Möglicherweise rührt dies von ihren wenig empfindlichen sensitometrischen Hilfsmitteln und den ebenso wenig wirksamen Luftpumpen (Min.-Druck etwa $6 \mathrm{~mm}$ ) her. Vnukova ${ }^{6}$ hat dagegen angegeben, daß der Herschel-Effekt durch Rotbelichtung in Sauerstoff intensiver als in Luft sein soll, was mit unserer Beobachtung in Einklang steht. Die Rich-

5 M. B la u u. H. W a m b a cher, Nature [London] 134, 538 [1934]; S.-B. Akad. Wiss. Wien 143, 285-301 [1934]; Photogr. Korresp. 1934, 31-40; Z. wiss. Photogr., Photophysik Photochem. 33, 191-197 [1934]; 34, 253 -266 [1935]; M. B l a u, Photogr. Korresp. 1935, 21-28. tigkeit dieses Resultats wurde aber etwas später von Thurston und Blair ${ }^{7}$ bestritten. Sie arbeiteten mit Gasen von gewöhnlichem Luftdruck, und es scheint nicht sicher, ob die Luft vor dem Einlaß des neuen Gases vollkommen entfernt war; auch benutzten sie gelbe Lichtfilter, die wohl nicht genügend zuverlässig sind. Wie wir a. a. ${ }^{8}{ }^{8}$ mitgeteilt haben, sind die Forderungen an das Filter bei Herschel-Beleuchtungen sehr streng.

Nun werden die Verhältnisse durch den von uns gefundenen Einfluß der Luft auf den Stabilisierungseffekt des photographischen Materials nach der Exposition etwas komplizierter. Der Herschel-Effekt und der Stabilisierungseffekt werden in der gleichen Richtung und durch denselben Mechanismus beeinflußt. In unseren eben genannten Versuchen sind die beiden Effekte nicht separiert. Die Blauexponierung erfolgte freilich in vollkommen ähnlicher Weise für die Filmproben A, C und D, die Stabilisierungsperiode dagegen verlief für $\mathrm{A}$ und $\mathrm{C}$ in Luft, für $\mathrm{D}$ hauptsächlich in Vakuum und in Stickstoff. Die Behandlung der Filmstreifen D ähnelte also mehr der Behandlung der Streifen B als der Streifen A. Die Schwärzungsdifferenz B-D ist im Maximum 1,76, also wenig verschieden von der Schwärzungsdifferenz 2,03 bei A-C, und vielleicht würde sie sich noch mehr dem letzten Wert nähern, falls das Evakuieren der Schicht schneller vor sich gehen könnte. Es scheint also denkbar, daß der Sauerstoff der Luft den Stabilisierungseffekt mehr beeinflußt als den Herschel-Effekt. Jedenfalls werden wir die Verhältnisse bei dem HerschelEffekt noch näher studieren.

In diesem Frühjahr haben wir die alten Versuche wieder aufgenommen. Wir haben dabei bis jetzt nur den Einfluß der Luft bei gewöhnlicher Exponierung, nicht aber beim Herscheln, untersucht. Statt dessen haben wir den Effekt sowohl bei Kornflächen-Entwicklung als bei Korntiefe-Entwicklung verfolgt.

In Abb. 2 sehen wir zwei typische Kurven für Kodak Process Plate B 20 bei Kornflächen-Entwicklung. Die Kurve A bezieht sich auf Exponierungen (weißes Licht) in Luft gewöhnlichen Druckes, Kurve B auf Exponierungen in Vakuum. Wie wir erwartet hatten, liegt die Kurve B im allgemeinen höher als A, was mit unserer Arbeitshypothese in Einklang steht.

6 A. Vnuk ova, Nature [London] 138, 246-247 [1936].

7 M. Thurston u. J. M. Blair, J. opt. Soc. America 28, September 1938.

8 H. B ä ck s t röm u. J. B a s be r g, J. opt. Soc. America 37, 891-898 [1947]. 
Es ist aber auch deutlich, daß bei höheren Dichten (über $D=2,75$ ) das Gegenteil eintritt. Soweit wir bisher gefunden haben, scheint dies bei kornflächenentwickelten Schichten allgemein der Fall zu sein.

Kodak Rapid Panchromatic P 300 zeigte ähnliche Verhältnisse sowohl bei Expositionen durch Orangefilter als durch Blaufilter. Im ersten Falle schnitten die Kurven einander etwa bei der Dichte 2,0, in dem zweiten Falle bei $D=2,5$. - Bei Gevaert-ReplicaFilm fielen die beiden Kurven in ihrem unteren Teil zusammen, trennten sich aber bei $D \approx 1,5$, wobei die Kurve B unter die Kurve A sank.

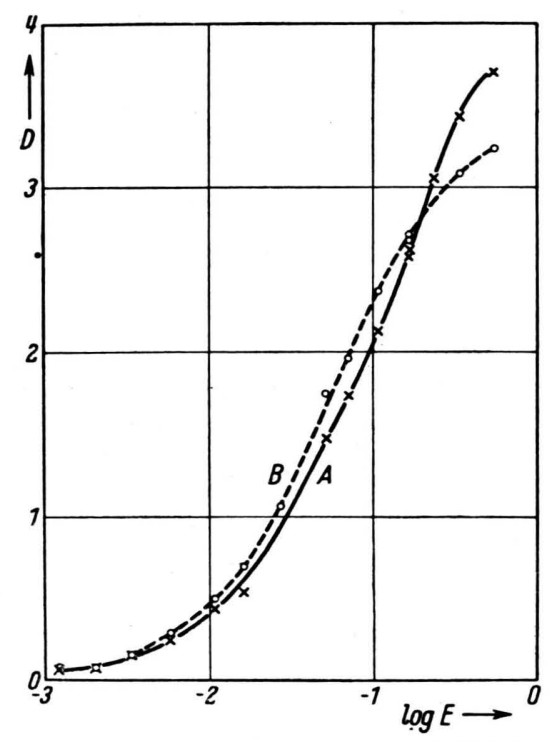

Abb. 2. Schwärzungskurven für Kodak-Process-Plate, kornflächenentwickelt. Die Exponierungen erfolgten für die beiden Kurven gleich, nur daß sich die Filmproben bei A in Luft, bei B im Vakuum befanden.

Wir haben auch einen Ausnahmefall gefunden, wo die Kurve B während ihres ganzen Verlaufs unterhalb der Kurve A liegt. Das gilt für Ilford-ProcessFilm P 40 (Abb. 3). Wir haben dieses anomale Verhalten durch mehrmalige Versuche festgestellt. Es ist übrigens dabei charakteristisch, daß die beiden Kurven wenigstens bis $D=4$ steil verlaufen und sich nur allmählich voneinander trennen, daß aber die - Kurve B nicht bei höheren Dichten umbiegt.

Bei der Korntiefe-Entwicklung scheinen die Verhältnisse sehr regelmäßig zu sein. Die Kurve B liegt höher als die Kurve A, und der Abstand zwischen ihnen ist sehr groß. Als Beispiel werden in Abb. 4 die Verhältnisse bei Kodak-Rapid-Panchromatic-Film, durch Orangefilter exponiert, wiedergegeben. Auch bei Ilford-Process-Film, der bei Kornflächenentwick- lung eine Ausnahme bildet, sind die Verhältnisse ähnlich. Nur Gevaert-Replica-Film zeigte zusammenlaufende Kurven. Charakteristisch war aber auch hier, daß bei hohen Dichten die B-Kurve sich über die A-Kurve hob.

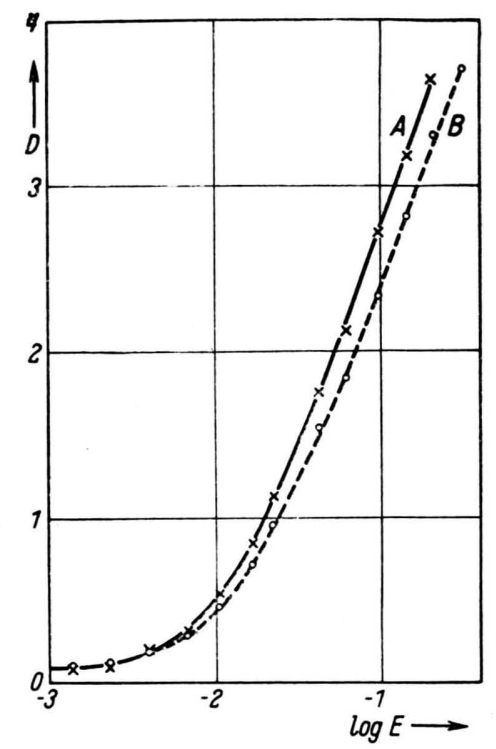

Abb. 3. Schwärzungskurven für kornflächenentwickelten Ilford-Process - Film, in Luft (Kurve A) und im Vakuum (Kurve B) exponiert. In diesem Falle liegt Kurve B unter der Kurve A.

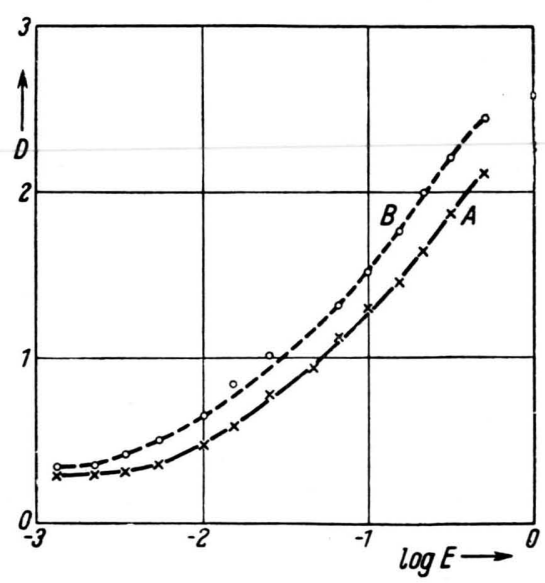

Abb. 4. Schwärzungskurven für Kodak-Rapid-Panchromatic-Film, durch Orangefilter exponiert und korntiefeentwickelt. Bei A wurden die Filmproben in Luft, bei B im Vakuum exponiert.

Unsere Untersuchungen über den Einfluß von Luft auf den photographischen Prozeß befinden sich erst im Anfangsstadium. Es scheint aber aus diesen vorläufigen Versuchen hervorzugehen, daß eine deutliche Einwirkung hauptsächlich in solcher Richtung geht, daß die Luft in der Schicht eine Erniedrigung 
der Empfindlichkeit mit sich bringt, die aber durch das Evakuieren der Schicht aufgehoben werden kann. Bei Korntiefe-Entwicklung scheint der Effekt im allgemeinen ziemlich groß auszufallen.

Von Interesse scheint weiter zu sein, daß die Einwirkung der Luft auch während der Stabilisierungsperiode nach der Exposition deutlich merkbar sein kann. Möglicherweise übt die Luft außerdem einen Einfluß auf den Herschel-Effekt aus, auch wenn kein Desensibilisator anwesend ist. Diese Verhältnisse werden wir aber später eingehender untersuchen.
Soweit wir wissen, hat man bisher keinen Einfluß der Luft auf die nach einer Exposition unter gewöhnlichen Umständen erhaltenen Schwärzungen gefunden. Wenigstens haben B la u und $\mathrm{Wa} \mathrm{mb}$ a c he $\mathrm{r}^{5}$, die sich mit Lufteinflüssen eingehend beschäftigt haben, mehrmals erklärt, daß sie eine solche Einwirkung nur bei desensibilisierten Schichten bemerkt haben. Unseres Erachtens muß aber der Effekt in diesem Fall größer werden. Er dürfte aber denselben Grund haben und möglicherweise von Brombildung an der Kornfläche hervorgerufen werden.

\title{
Über die Silberausscheidung durch Keime beim Silbersalzdiffusionsverfahren (Agfa-Copyrapidprozess)
}

\author{
Von Edith Weyde \\ Aus dem wissenschaftlichen Laboratorium der Agfa-Photofabrik, Leverkusen-Bayerwerk
}

(Z. Naturforschg. 6 a, 381-382 [1951]; eingegangen am 4. April 1951)

Herrn Professor Dr. John Eggert zum 60. Geburtstage gewidmet

$\mathrm{D}$ ie neuen Silbersalzdiffusionsverfahren, deren wichtigstes Anwendungsgebiet in der schnellen Herstellung direkter Positive liegt, kann man als eine Modifikation der physikalischen Entwicklung ansehen. Bei dieser beschleunigen bekanntlich die durch Licht entstandenen Silberkeime nach Herauslösen des Halogensilbers die Reduktion gelöster Silbersalze (physikalischer Entwickler), wobei man ein negatives Silberbild erhält. Bei den Silbersalzdiffusionsverfahren enthält dagegen die Schicht, in der das Positiv entstehen soll, von vornherein die Keime, und zwar gleichmäßig verteilt. Zur Bildentstehung kommt es, wenn man diese Positivschicht mit einer belichteten Halogensilberschicht - der Negativschicht - in engen Kontakt bringt, nachdem man beide Schichten mit einem photographischen Entwickler durchfeuchtet hat, der ein Halogensilberlösungsmittel, wie z. B. Natriumthiosulfat enthält. Die belichteten Halogensilberkörner der Negativschicht werden dabei zu Silber reduziert, während sich das unbelichtete Halogensilber auflöst. Diese in der Negativschicht gebildeten löslichen Silbersalze muß man dazu zwingen, zum Großteil in die Positivschicht abzuwandern, damit man dort ein genügend gedecktes Positiv erhält. Dies erreicht man am besten durch eine rasche Reduktion der Silbersalze in der Positivschicht, wodurch das Konzentrationsgefälle in bezug auf die gelösten Sil-- bersalze zwischen den beiden Schichten während der Entstehung des Positivs groß bleibt. Auch in der Negativschicht sind Keime vorhanden, die die Reduktion der Silbersalze beschleunigen: an den mehr oder minder stark belichteten Stellen entwickeltes Silber, an den unbelichteten Stellen immer geringe Mengen Schleiersilber.

Nach den Arbeiten von E g g e r t und A re n s ${ }^{1}$ ist aber für die Schnelligkeit der Reduktion der Silbersalze nicht die absolute Menge des Silbers, sondern nur die Anzahl der Silberkeime maßgebend. Danach muß man also bei den Silbersalzdiffusionsverfahren einerseits darauf hinarbeiten, der Positivschicht möglichst viel Keime einzuverleiben, so daß deren Anzahl in der Negativschicht keine Rolle spielt, andererseits muß die Menge des Keimsilbers in der Positivschicht sehr klein bleiben, da diese nicht angefärbt werden darf. Eine sehr schnelle Reduktion der gelösten Silbersalze in der Positivschicht ist übrigens auch notwendig, um scharfe Bilder zu erhalten, denn nur so kann man die seitliche Diffusion der Silbersalze beschränken.

Für den speziellen Fall des Agfa-Copyrapid-Verfahrens ${ }^{2}$, das bekanntlich zur schnellen Herstellung

1 J. Eg g e rt, Helv. chim. Acta 30, 1750 [1947]; H. Arens, Agfa-Veröffentlichungen III, S. 32 [1933] (daselbst siehe weitere Literaturangaben); J. E g g e r t u. H. A re n s, Z. Elektrochem. angew. physik. Chem. 35, 728 [1929]. 